\title{
The Continuous Hexachordal Theorem
}

\author{
Brad Ballinger ${ }^{1}$, Nadia Benbernou ${ }^{2}$, Francisco Gomez ${ }^{3}$, Joseph O'Rourke ${ }^{4}$, \\ and Godfried Toussaint ${ }^{5}$ \\ ${ }^{1}$ Davis School for Independent Study, Davis, USA \\ ${ }^{2}$ Department of Mathematics and Computer Science and Artificial Intelligence \\ Laboratory (CSAIL), Massachusetts Institute of Technology, Cambridge, USA \\ ${ }^{3}$ Department of Applied Mathematics, Universidad Politecnica de Madrid, \\ Madrid, Spain \\ ${ }^{4}$ Department of Computer Science, Smith College, Northampton, USA \\ ${ }^{5}$ Centre for Interdisciplinary Research in Music Media and Technology, \\ The Schulich School of Music, McGill University, Montreal, Canada
}

\begin{abstract}
The Hexachordal Theorem may be interpreted in terms of scales, or rhythms, or as abstract mathematics. In terms of scales it claims that the complement of a chord that uses half the pitches of a scale is homometric to - i.e., has the same interval structure as - the original chord. In terms of onsets it claims that the complement of a rhythm with the same number of beats as rests is homometric to the original rhythm. We generalize the theorem in two directions: from points on a discrete circle (the mathematical model encompassing both scales and rhythms) to a continuous domain, and simultaneously from the discrete presence or absence of a pitch/onset to a continuous strength or weight of that pitch/onset. Athough this is a significant generalization of the Hexachordal Theorem, having all discrete versions as corollaries, our proof is arguably simpler than some that have appeared in the literature.

We also establish the natural analog of what is sometimes known as Patterson's second theorem: if two equal-weight rhythms are homometric, so are their complements.
\end{abstract}

\section{Introduction}

\subsection{Basic Definitions}

We are concerned with cyclic musical rhythms consisting of $k$ onsets (pulses, beats) and $n-k$ rests, represented by $n$ evenly spaced points on a circle, with arithmetic $\bmod n$, i.e., in the group $\mathbb{Z}_{n}$. This representation has been used as early as the 13th century, as accounted by Wright Wri78], but it has been used recently again; see Vuz85], Tou05], among others. Alternately, the $k$ onsets (points) may be considered as $k$ pitches making up a musical chord or scale selected from a universe of $n$ pitches [ym06]. Such sets of points on a circle are called cyclotomic sets in the crystallography literature [Pat44], Bue78]. We will emphasize the rhythms model in this paper, but all results hold equally in the pitch model or the crystallography model.

E. Chew, A. Childs, and C.-H. Chuan (Eds.): MCM 2009, CCIS 38, pp. 11-21 2009.

(C) Springer-Verlag Berlin Heidelberg 2009 
Every pair of the points on the circle determines an inter-onset duration interval (the geodesic between the pair of points around the circle) Bue78]. The histogram of this multiset of distances in the context of musical scales and chords is called its interval content Lew59]. Two rhythms which are congruent to each other obviously have the same interval content. Here by congruence we mean geometrical congruence, i.e., equivalence under rotation or reflection. However, two rhythms with the same histograms need not be congruent. Two sets of points with the same multiset of distances are said to be homometric, a term introduced by Patterson in 1939 [Pat44], who first discovered them. In the music literature, two pitch-class sets (or two rhythms) with the same intervalic content are termed as having the $Z$-relation or isomeric relation [For77].

One of the fundamental theorems in this area is the so-called Hexachordal Theorem, which states that complementary sets with $k=n / 2$ (and $n$ even) are homometric. Two examples are shown in Figs. 1 and 2, In Fig. 1, the $k=4$ onsets occur at $(0,1,4,7)$, and the complementary rhythm has onsets precisely where the first rhythm has rests: $(2,3,5,6)$. The histogram of intervals is identical.

Fig. 2 shows two complementary $(n, k)=(12,6)$ rhythms, again with identical histograms.

An important convention we follow is that the pair of onsets separated by the diameter $d=n / 2$ contributes two counts to the interval $d$ in the histogram. This
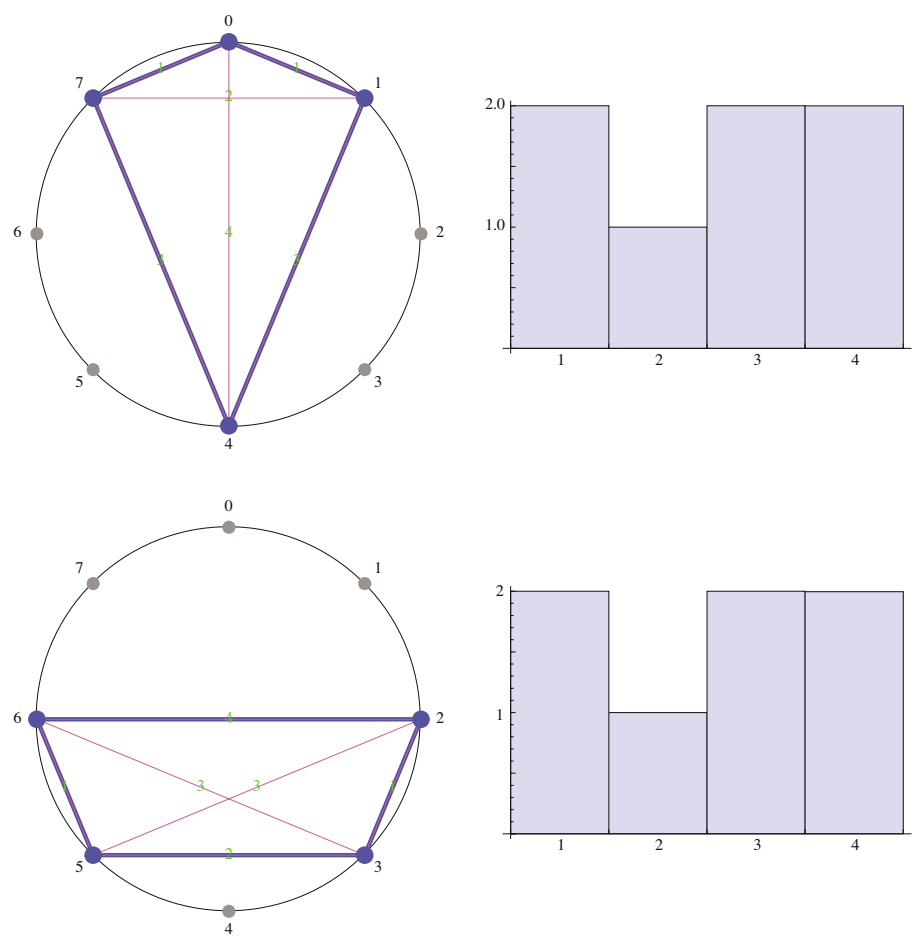

Fig. 1. Example of the Hexachordal Theorem, $(n, k)=(8,4)$. Note that the distance $d=4$ is counted twice. 

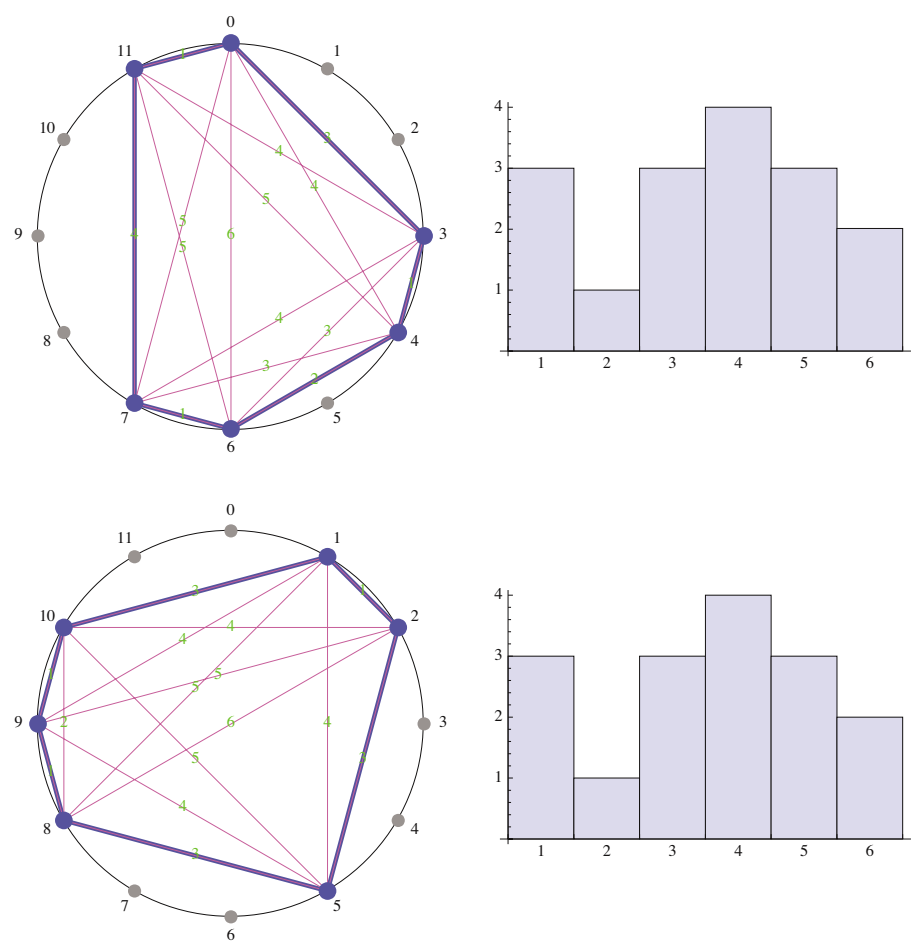

Fig. 2. Another example of the Hexachordal Theorem, $(n, k)=(12,6)$. Note that the distance $d=6$ is counted twice.

convention simplifies the proofs but changes nothing substantively. This issue is further addressed in Section 2.5,

The term "hexachordal" derives from Schönberg's use of 6-note chords in a 12-tone chromatic scale, and the name "hexachordal" has been retained even though the theorem holds for arbitrary even $n$.

\subsection{History}

The earliest proof of the Hexachordal Theorem in the music literature is, to our knowledge, due to Lewin. In 1959 he published a paper [Lew59] on the intervalic relations of two chords that contained an embryonic proof of the Hexachordal Theorem; such a proof was refined in a subsequent paper [Lew60]. In 1974 Regener [Reg74] found an elementary simple proof of this theorem based on the combinatorics of pitch-class sets. Many other proofs have appeared since then, often rivalling in conciseness. Short proofs can be found, for instance, in the work of Mazzola [Maz03] or Jedrzejewski Jed06]. Amiot [Ami07] gave an elegant, short proof based on the discrete Fourier transform. Perhaps, one of the simplest proofs, in the sense of using no structures such as groups or discrete Fourier transforms, was discovered by Blau [Bla99]. His proof relies on a straight- 
Theorem 1. If $R$ is a rhythm on $n$ points, $n$ even, and $W(R)=n / 2$, then $R$ and $\bar{R}$ are homometric: for all distances $d, H_{\bar{R}}(d)=H_{R}(d)$.

Before proceeding to the continuous domain, we need Lemma 1 below, which expresses the histogram function in terms of the weights. This lemma is known in the music literature as the "common-tone theorem" [Joh03]. See JK06] for a proof in the context of group theory. For the sake of completeness, we include our own proof.

Lemma 1. $H_{R}(d)=\sum_{i=0}^{n-1} w_{i} w_{i+d}$.

Proof. Point $i$ is separated by a distance $d$ from the point at $i+d$, where we interpret addition $\bmod n$, i.e., in $\mathbb{Z}_{n}$. If both are onsets, then $w_{i}=w_{i+d}=1$, and $w_{i} w_{i+d}=1$. If either point is a rest, then $w_{i} w_{i+d}=0$. Thus, for each fixed $d$, summing $w_{i} w_{i+d}$ over all $i$ counts 1 for each occurrence of $d$.

We now argue that each pair of points realizing a distance $d$ contributes just once to the sum. A pair $(i, i+d)$ would contribute twice if $i+2 d=i$ so that $(i+d, i)$ would be counted as well. Because $d$ is a shortest path, we have $d \leq n / 2$. Thus, $i+2 d \leq i+n$, and this equals $i$ (in $\mathbb{Z}_{n}$ ) only when $d=n / 2$ is the diameter. Our convention is indeed to count a pair realizing the diameter twice.

Consider, for example, the $n=12$ example in Fig. 2 (top). For $d=n / 2=6$, both $w_{0} w_{6}$ and $w_{6} w_{12}=w_{6} w_{0}$ contribute to $H_{R}(6)=2$. Indeed, the reason we follow the convention of double-counting each realization of the diameter is that it naturally fits this weight viewpoint. This point will be revisited in Section 2.5.

\subsection{The Continuous Generalizations}

We generalize in two directions. First, the circle of $n$ discrete points is generalized to a continuous circle of points. We take its circumference to be 1 without loss of generality. Second, the discrete set of weights $w_{i}$ is generalized to a real-number weight $f(x) \in[0,1]$ for $x \in[0,1]$. Here $x$ specifies a point on the circle, measured by distance clockwise from the zero-position (conventionally at the 12 o'clock position as in Figures (1) and 2), and $f(x)$ the weight of that point. So now the total weight $W(R)=\int_{0}^{1} f(x) d x$. Note the maximum possible total weight of any rhythm is achieved by the constant "rhythm" with weight $f(x)=1$ for all $x$, in which case $W(R)=1$.

We define the complement of a rhythm analogously to the discrete case:

Definition 1. For each point $x$ in rhythm $R$ with weight $f(x)$, the corresponding point $x$ in the complementary rhythm $\bar{R}$ has weight $\overline{f(x)}=1-f(x)$.

The histogram $H_{R}(d)$ is generalized to a function over the domain $d \in\left[0, \frac{1}{2}\right]$. We need the continuous analog of Lemma 1 In fact, we take the analog of that lemma as the definition of the histogram in the continuous domain:

Definition 2. $H_{R}(d)=\int_{0}^{1} f(x) f(x+d) d x$.

For example, if two points $x$ and $x+d$ each have weight $\frac{1}{2}$, they contribute $\frac{1}{4}$ to the height of $H_{R}$ at distance $d$. 


\subsection{Continuous Hexachordal Theorem and Proof}

The Continuous Hexachordal Theorem says that for any rhythm on the continuous circle as described above, if the rhythm has weight $\frac{1}{2}$, then it is homometric to its complement. More formally, it may be stated as:

Theorem 2. If $R$ is a integrable rhythm on the continuous circle, and $W(R)=$ $\frac{1}{2}$, then for all distances $d, H_{\bar{R}}(d)=H_{R}(d)$.

Proof. The proof fixes $d$ and establishes that $H_{\bar{R}}(d)=H_{R}(d)$. From the histogram Definition 2 we have:

$$
H_{\bar{R}}(d)=\int_{0}^{1} \overline{f(x)} \overline{f(x+d)} d x .
$$

From the complement Definition 1 this is:

$$
=\int_{0}^{1}[1-f(x)][1-f(x+d)] d x .
$$

Multiplying out terms yields:

$$
=\int_{0}^{1}(1-f(x)-f(x+d)+f(x) f(x+d)) d x .
$$

Separating integrals gives:

$$
=\int_{0}^{1} 1 d x-\int_{0}^{1} f(x) d x-\int_{0}^{1} f(x+d) d x+\int_{0}^{1} f(x) f(x+d) d x
$$

The first integral is just 1 , and the second twd 1 are each $\frac{1}{2}$ by the assumption of the theorem that $W(R)=\frac{1}{2}$ :

$$
\begin{aligned}
& =1-\frac{1}{2}-\frac{1}{2}+\int_{0}^{1} f(x) f(x+d) d x \\
& =\int_{0}^{1} f(x) f(x+d) d x \\
& =H_{R}(d)
\end{aligned}
$$

The last step again follows from the Definition 2, and so we have established that $H_{\bar{R}}(d)=H_{R}(d)$ for all $d$, i.e., the histograms are identical and $R$ is homometric to $\bar{R}$.

The weight function $f(x)$ need not be a continuous function in the technical mathematical sense 2 We only need that it be integrable 3 i.e., a function for which an appropriate "area under the function graph" may be defined.

\footnotetext{
${ }^{1}$ Shifting $x$ to $x+d$ shifts the graph of $f($ ) but does not change the area underneath it.

${ }^{2}$ A function $f$ is continuous if, for all $c$ in the domain, $\lim _{x \rightarrow c} f(x)=f(c)$.

${ }^{3}$ For example, Lebesgue integrable suffices.
} 
(a)

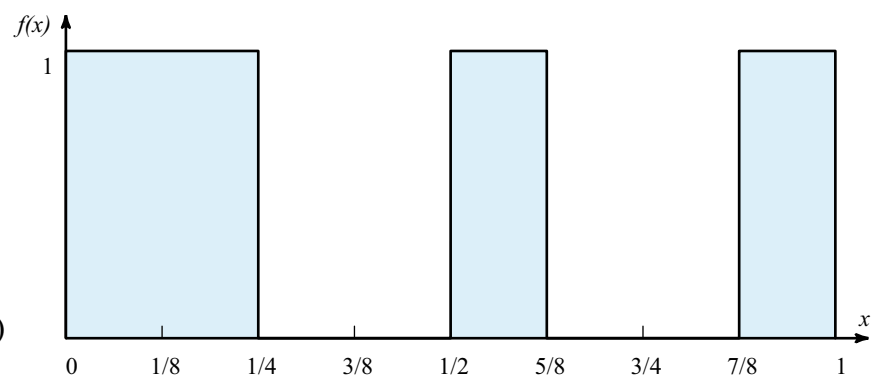

(b)

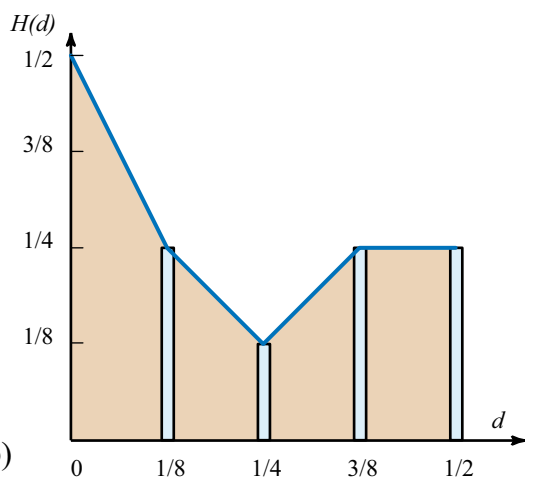

Fig. 3. (a) Weight step function $f(x)$ corresponding to Fig. 1 (top), $(n, k)=(8,4)$. (b) Corresponding histogram integral $H(d)$.

We should note that the above proof can be directly discretized to yield a parallel proof of the Discrete Hexachordal Theorem. Instead, we show below that the freedom to use any integrable weight function renders the Discrete Hexachordal Theorem 1 an immediate corollary of the Continuous Hexachordal Theorem 2 ,

\subsection{Discrete Theorem as Corollary}

Suppose a discrete rhythm $R$ has weights $\left(w_{0}, w_{1}, \ldots, w_{n-1}\right)$, with each weight either 1 or 0 . Then define the step function $f(x)=w_{i}$ for $\frac{i}{n} \leq x<\frac{i+1}{n}$. For example, Fig. 3(a) shows the step function corresponding to the top rhythm in Fig. 11 whose discrete weights are $(1,1,0,0,1,0,0,1)$. Note that the total weight/area is $4 \cdot \frac{1}{8}=\frac{1}{2}$, which accords with the discrete weight of $\frac{1}{2} n=\frac{1}{2} 8=4$.

We formalize this correspondence between continuous and discrete as follows:

Corollary 1. The Discrete Hexachordal Theorem 1 follows from the Continuous Hexachordal Theorem [2.

Proof. We use the notation 


$$
\chi_{A}(x)=\left\{\begin{array}{l}
1, \text { for all } x \in A \\
0, \text { otherwise }
\end{array}\right.
$$

to represent the $1 / 0$ characteristic function of a set $A$.

We convert the discrete rhythm $\left(w_{0}, w_{1}, \ldots, w_{n-1}\right)$ into the continuous rhythm

$$
f(x)=\sum_{i=0}^{n-1}\left(w_{i} \cdot \chi_{\left[\frac{i}{n}, \frac{i+1}{n}\right)}\right) .
$$

This has the feature, mentioned above, that for all $x \in\left[\frac{i}{n}, \frac{i+1}{n}\right)$, we have $f(x)=w_{i}$.

Because of the horizontal compression involved in this conversion, the discrete histogram contribution $H_{R}(d)=\sum_{i=0}^{n-1} w_{i} w_{i+d}$ corresponds to the continuous histogram contribution

$$
\begin{aligned}
& H_{R}\left(\frac{d}{n}\right)=\int_{0}^{1} f(x) f\left(x+\frac{d}{n}\right) d x \\
& =\int_{0}^{1}\left[\sum_{i=0}^{n-1}\left(w_{i} \cdot \chi_{\left[\frac{i}{n}, \frac{i+1}{n}\right)}\right)\right] f\left(x+\frac{d}{n}\right) d x \\
& =\sum_{i=0}^{n-1}\left[w_{i} \int_{0}^{1} \chi_{\left[\frac{i}{n}, \frac{i+1}{n}\right)} \cdot f\left(x+\frac{d}{n}\right) d x\right] \\
& =\sum_{i=0}^{n-1}\left[w_{i} \int_{\frac{i}{n}}^{\frac{i+1}{n}} f\left(x+\frac{d}{n}\right) d x\right] \\
& =\sum_{i=0}^{n-1}\left[w_{i} \int_{\frac{i+d}{n}}^{\frac{i+d+1}{n}} f(x) d x\right] \\
& =\sum_{i=0}^{n-1}\left[w_{i} \int_{\frac{i+d}{n}}^{\frac{i+d+1}{n}} w_{i+d} d x\right] \\
& =\frac{1}{n} \sum_{i=0}^{n-1} w_{i} w_{i+d}
\end{aligned}
$$

So, the continuous histogram is proportional to the discrete histogram at integral values of $d$ (see Fig. 3(b)), and the conclusion of the Continuous Hexachordal Theorem 2 that $R$ is homometric to $\bar{R}$ implies the same in the discrete case, which is precisely the claim of the Discrete Hexachordal Theorem 1 .

\subsection{Double-Counting Diameter Intervals}

We return to the the issue of double-counting an interval that equals the diameter $\left(d=n / 2\right.$ in the discrete case or $d=\frac{1}{2}$ in the continuous case $)$ in the histogram $H_{R}(d)$. In music the diameter in the case of an equal-temperament 
scale corresponds to a tritone. Recall from Definition 2 that the continuous histogram is defined by the equation $H_{R}(d)=\int_{0}^{1} f(x) f(x+d) d x$. Applying this for $d=\frac{1}{2}$ to the step function $f(x)$ in Figure 3 results in

$$
H_{R}\left(\frac{1}{2}\right)=\int_{0}^{1} f(x) f\left(x+\frac{1}{2}\right) d x
$$

When $x \in\left[0, \frac{1}{8}\right)$, the product $f(x) f\left(x+\frac{1}{2}\right)$ is 1 . And also when $x \in\left[\frac{1}{2}, \frac{5}{8}\right)$, the product is again 1 , because $x+\frac{1}{2}$ wraps around to $\left[0, \frac{1}{8}\right)$. For all other $x$, the product is 0 . So $H_{R}\left(\frac{1}{2}\right)=2 \cdot \frac{1}{8}=\frac{1}{4}$, which corresponds to the height 2 for $d=4$ in the discrete case in Figure 1] Thus, the continuous histogram analog also "double-counts" the diameter $d=\frac{1}{2}$.

Moreover, we can see that this is the natural definition, by considering $d=$ $\frac{1}{2}-\varepsilon$ for some small $\varepsilon>0$. The same integral leads to $H_{R}\left(\frac{1}{2}-\varepsilon\right)=2\left(\frac{1}{8}-\varepsilon\right)$ which goes to $\frac{1}{4}$ as $\varepsilon \rightarrow 0$. Thus, the height $H_{R}\left(\frac{1}{2}\right)$ is consistent with the limit for $d<\frac{1}{2}$. Stipulating that $d=\frac{1}{2}$ should be treated specially would destroy this natural correspondence.

\subsection{Patterson's First Theorem}

Patterson's first Theorem [Pat44] goes beyond the $k=n / 2$ precondition of the Discrete Hexachordal Theorem 1 It may be stated as: two homometric $(n, k)$ rhythms have homometric complements. In our continuous generalizations, two rhythms with the same number $k$ of onsets have the same weight. So the generalization is:

Theorem 3. If $R_{1}$ and $R_{2}$ are two integrable rhythms on the continuous circle with equal weights, $W\left(R_{1}\right)=W\left(R_{2}\right)$, and they are homometric, i.e., for all distances $d, H_{R_{1}}(d)=H_{R_{2}}(d)$, then their complements are homometric: $H_{\overline{R_{1}}}(d)=H_{\overline{R_{2}}}(d)$.

Proof. Let the weight function of $R_{1}$ be $f(x)$ and that of $R_{2}$ be $g(x)$. Fix a distance $d$. We compute $H_{\overline{R_{1}}}(d)$ and show it is equal to $H_{\overline{R_{2}}}(d)$. From Definitions 2 and 1, we have

$$
\begin{aligned}
H_{\overline{R_{1}}}(d) & =\int_{0}^{1} \overline{f(x)} \overline{f(x+d)} d x \\
& =\int_{0}^{1}(1-f(x))(1-f(x+d)) d x
\end{aligned}
$$

Multiplying out terms and separating integrals yields

$$
\begin{aligned}
& =\int_{0}^{1} 1 d x-2 \int_{0}^{1} f(x) d x+\int_{0}^{1} f(x) f(x+d) d x \\
& =1-2 \int_{0}^{1} f(x) d x+\int_{0}^{1} f(x) f(x+d) d x
\end{aligned}
$$


Now, because $W\left(R_{1}\right)=W\left(R_{2}\right)$, we have $\int_{0}^{1} f(x) d x=\int_{0}^{1} g(x) d x$, and because $R_{1}$ and $R_{2}$ are homometric, we have $\int_{0}^{1} f(x) f(x+d) d x=\int_{0}^{1} g(x) g(x+d) d x$ :

$$
=1-2 \int_{0}^{1} g(x) d x+\int_{0}^{1} g(x) g(x+d) d x
$$

However, we know, by the same reasoning, that this expression is

$$
=\int_{0}^{1} \overline{g(x)} \overline{g(x+c)} d x
$$

And we have therefore established that the complementary rhythms are homometric:

$$
\begin{aligned}
\int_{0}^{1} \overline{f(x)} \overline{f(x+d)} d x & =\int_{0}^{1} \overline{g(x)} \overline{g(x+d)} d x \\
H_{\overline{R_{1}}}(d) & =H_{\overline{R_{2}}}(d)
\end{aligned}
$$

\section{Open Problems}

Our results may be interpreted in terms of polyphonic rhythms, in which several instruments are linearly combined [OTT08]. For instance, to model three identical drums playing together, interpret the weight $f(x)=\frac{1}{3}$ to mean that one drum is struck on a particular beat, while the weight $f(x)=1$ would mean all three are struck. It would be interesting to explore whether homometric polyphonic rhythms have a musical significance.

We know that two sets of points with different cardinalities and different weights may be homometric, but we neither understand the constraints here mathematically nor know if there is any musical interpretation of such sets.

Theorem 2 generalizes to weights in $[0,1]$ on a sphere, with distances measured by geodesics, and with $W(R)=\frac{1}{2}$ corresponding to the integral over a hemisphere equalling $\frac{1}{2}$. The discrete analog is "distance regular" points on a sphere, e.g., the vertices of a Platonic solid. Is there any musical analog for spheres in any dimension?

\section{Acknowledgements}

The authors like to thank the anonymous referees for their useful comments.

\section{References}

[AG00] Althuis, T.A., Göbel, F.: Z-related pairs in microtonal systems. Memorandum 1524, University of Twente, The Netherlands (April 2000)

[Ami07] Amiot, E.: David Lewin and maximally even sets. Journal of Mathematics and Music 1(3), 157-172 (2007)

[Bla99] Blau, S.K.: The hexachordal theorem: A mathematical look at interval relations in twelve-tone composition. Mathematics Magazine 72(4), 310-313 (1999) 
[Bue76] Buerger, M.J.: Proofs and generalizations of Patterson's theorems on homometric complementary sets. Zeitschrift für Kristallographie 143, 79-98 (1975)

[Bue78] Buerger, M.J.: Interpoint distances in cyclotomic sets. The Canadian Mineralogist 16, 301-314 (1978)

[For77] Forte, A.: The Structure of Atonal Music. The Yale University Press, Madison (1977)

[Igl81] Iglesias, J.E.: On Patterson's cyclotomic sets and how to count them. Zeitschrift für Kristallographie 156, 187-196 (1981)

[Jed06] Jedrzejewski, G.: Mathematical Theory of Music. Editions Delatour France (2006)

[JK06] Jaming, P., Kolountzakis, M.: Reconstruction of functions from their triple correlations. New York Journal of Mathematics 9, 149-164 (2003)

[Joh03] Johnson, T.: Foundations of Diatonic Theory. Key College Publishing (2003)

[Lew59] Lewin, D.: Intervallic relations betwen two collections of notes. Journal of Music Theory 3(2), 298-301 (1959)

[Lew60] Lewin, D.: The intervallic content of a collection of notes, intervallic relations between a collection of notes and its complement: An application to schoenberg's hexachordal pieces. Journal of Music Theory 4(1), 98-101 (1960)

[Lew76] Lewin, D.: On the interval content of invertible hexachords. Journal of Music Theory 20(2), 185-188 (Autumn 1976)

[Lew87] Lewin, D.: Generalized Musical Intervals and Transformations. Yale University Press (1987)

[Maz03] Mazzola, G.: The Topos of Music. Birkhäuser, Basel (2003)

[Mor90] Morris, R.D.: Pitch-class complementation and its generalizations. Journal of Music Theory 34(2), 175-245 (Autumn 1990)

[OTT08] O'Rourke, J., Taslakian, P., Toussaint, G.: A pumping lemma for homometric rhythms. In: Proc. 20th Canad. Conf. Comput. Geom., August 2008, pp. 99$102(2008)$

[Pat44] Lindo Patterson, A.: Ambiguities in the x-ray analysis of crystal structures. Physical Review 64(5-6), 195-201 (1944)

[Reg74] Regener, E.: On Allen Forte's theory of chords. Perspectives of New Music 13(1), 191-212 (Autumn-Winter 1974)

[Sen08] Senechal, M.: A point set puzzle revisited. European Journal of Combinatorics 29(1), 1933-1944 (2008)

[Sod95] Soderberg, S.: Z-related sets as dual inversions. Journal of Music Theory 39(1), 77-100 (Spring 1995)

[Tou05] Toussaint, G.T.: The geometry of musical rhythm. In: Akiyama, J., Kano, M., Tan, X. (eds.) JCDCG 2004. LNCS, vol. 3742, pp. 198-212. Springer, Heidelberg (2005)

[Tym06] Tymoczko, D.: The geometry of musical chords. Science 313(72), 72-74 (2006)

[Vuz85] Vuza, D.: Sur le rythme périodique. Revue Roumaine de Linguistique Cahiers de Linguistique Théorique et Appliquée 22(1), 103173-103188 (1985)

[Wri78] Wright, O.: The Modal System of Arab and Persian Music AD 1250-1300. Oxford University Press, Oxford (1978) 